\title{
80. On the Fluctuation of the Zone of Macroseismic Activity in the Pacific Ocean.
}

\author{
By Naomi MiYabe.
}

Earthquake Research Institute, Tokyo Imperial University.

(Rec. June 6, 1929. Comm. by T. Terada, M.I.A., June 12, 1929.)

In our previous investigation entitled "A Long Period Fluctuation in Latitude of the Seismic Activity on the Earth,"') the destructive earthquakes, of which the epicentres were located in the oceanic area, are excluded from our statistical data. In the present paper, those earthquakes are especially studied which originated in the domain of the Pacific Ocean. The data were taken, as before, from the chronological list of the destructive earthquakes given in the "Rika Nenpyo" compiled by Prof. A. Imamura. There were recorded 126 such destructive earthquakes during the recent twenty years, from 1906 to 1925, that are taken into account in the present investigation. Before entering into the statistical treatment, the position of the epicentre of each earthquake was referred to a new spherical coordinates, $a$ and $\zeta^{\prime \prime}$, the pole ${ }^{2)}$ of which is taken at $\varphi=0, \lambda=180^{\circ}$, where $\varphi$ is the latitude and $\lambda$ the longitude, such that

$$
\begin{aligned}
& \left.\cos a=\cos \varphi \cos \lambda_{1}{ }^{3}\right) \\
& \cos \phi^{\prime}=\cos \varphi \frac{\sin \lambda_{1}}{\sin a}
\end{aligned}
$$

Then, the whole region about the new pole is divided into the octants, ${ }^{\prime)}$ and mean values $a$ 's and $\psi^{\prime}$ 's respectively of all the earthquakes falling in each octant were calculated for successive epochs of five years. Points corresponding to these mean values are plotted in Fig. 1. Glancing at this figure, we may notice that there seems to exist a secular wandering of the zone of macroseismic activity, as far as we may infer from the scanty data. It may be pointed out that the active

1) T. Terada and N. Miyabe, Bull. Earthq. Res. Inst., 6 (1929), 333.

2) The pole is so selected, for convenience's sake, as the epicentres of the destructive earthquakes in these localities fall approximately in a zonal region around the Pacific Ocean.

3) Here, $\lambda_{1}=180^{\circ}-|\lambda|$, where $\lambda$ denotes the longitude. The positions of the epicentres are given in $\dot{\gamma}$ and $\rangle$, in the chronological list of the destructive earthquakes in the "Rika Nenpyo."

4) The octants are numbered counter-clockwise starting from E. (See Fig. 1.) 
zone in 4 th., 5th., and 6th. had been gradually wandering westwards in the epoch 1911-1925.

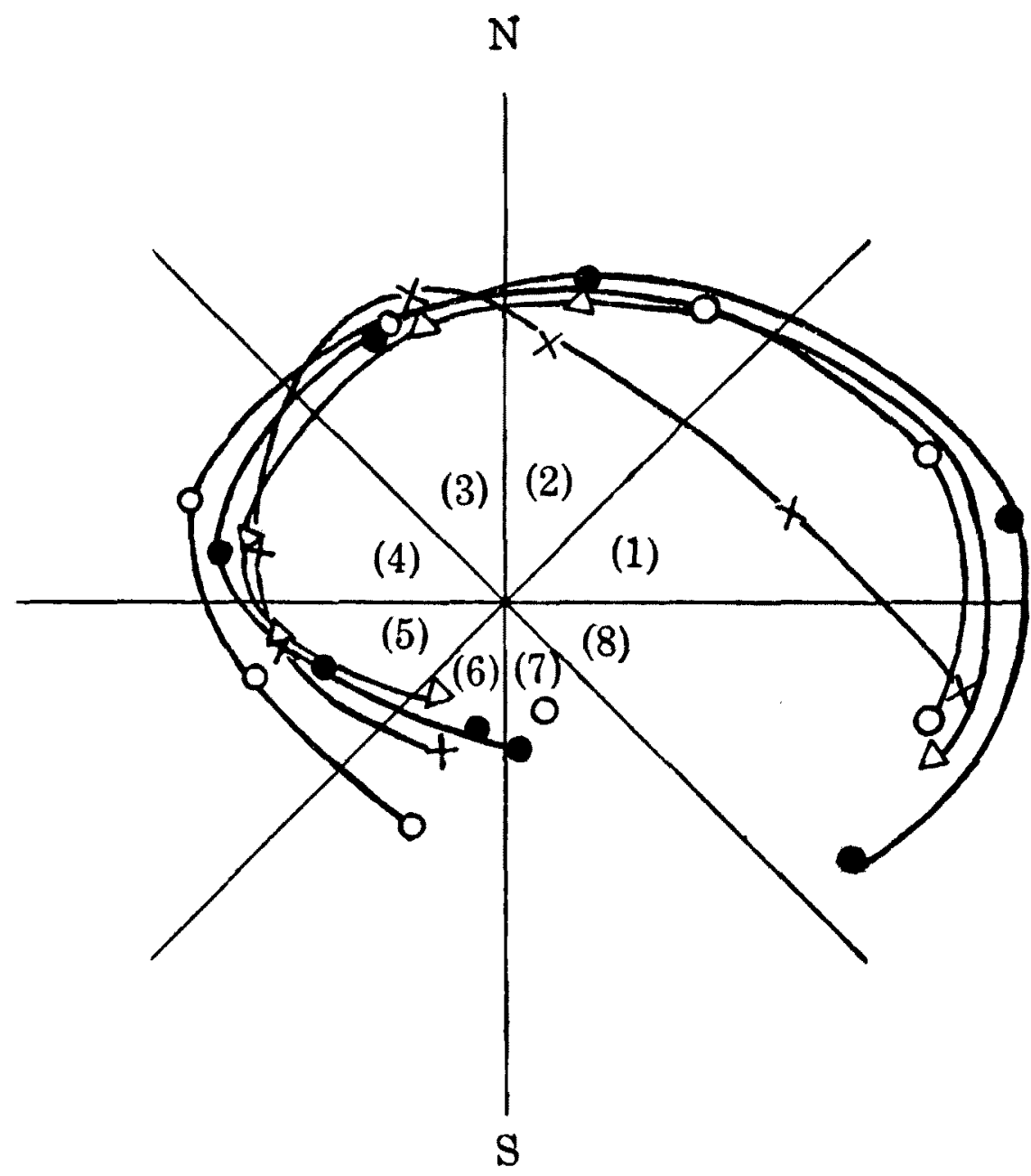

Fig. 2.

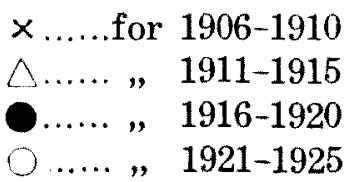

The frequencies of the earthquakes in these octants are given in Table I. The frequency maximum ${ }^{1)}$ may be seen fluctuating between 6th. and 4th. octants, where the epicentres of destructive earthquakes are most densely distributed. These regions seem, indeed, to be the most conspicuous sites of the seismic activity in the Pacific Ocean.

A comparison between the secular displacement of the active zone and that of the earth's axis of rotation suggests a certain correlation between the two phenomena. The more detailed description will appear

1) The frequency maximum occurs at 6th. octant in 1906-1910, at 4th. octant in 1911-1915, at 5th. octant in 1916-1920, and at 4th. octant in 1921-1925. 
No. 6.] On the Fluctuation of the Zone of Macroseismic Activity.

in Vol. VII, No. 2 of the Bulletin of the Earthquake Research Institute. The present writer expresses many thanks to Professor T. Terada for his kind advice and guidance.

Table I.

\begin{tabular}{|c|c|c|c|c|c|c|c|c|c|}
\hline Epoch & 1 & 2 & 3 & 4 & 5 & 6 & 7 & 8 & Sun \\
\hline $1906-1910$ & 3 & 1 & 3 & 4 & 3 & 8 & 0 & 1 & 23 \\
\hline $1911-1915$ & 0 & 3 & 4 & 7 & 1 & 1 & 0 & 2 & 18 \\
\hline $1916-1920$ & 1 & 1 & 7 & 8 & 12 & 3 & 6 & 2 & 40 \\
\hline $1921-1925$ & 3 & 3 & 9 & 10 & 6 & 6 & 2 & 6 & 45 \\
\hline $1906-1925$ & 7 & 8 & 23 & 29 & 22 & 18 & 8 & 11 & 126 \\
\hline
\end{tabular}

